\title{
A Rare Case of a Metastatic Basal Cell Carcinoma
}

\author{
Hiba Chaudhry, Dimitrios Doumpiotis
}

\section{ABSTRACT}

Background: Basal cell carcinomas are malignant slow-growing neoplasms of the epidermal layer of the skin. They are the most common form of skin cancer, accounting for $80 \%$ of non-melanoma skin cancers. Metastasis is rare with an incidence rate of 0.0028 to $0.55 \%$. We report a case of an 83 year old male presenting with basal cell carcinoma (BCC) of the right cheek. The tumour was completely excised with close margins. The patient was closely monitored clinically and on follow-up, a mass in the region of the right parotid was identified. Excision of the tail of the right parotid identified a tumour with similar subtype to the primary tumour excised from the cheek confirming diagnosis of metastatic BCC.

Methods: A literature search was carried out inclusive of the terms identify existing data and guidelines to determine best evidence based practice.

Discussion: Literature search identified aetiology of BCCs and methods of diagnosis and treatment. The search also highlighted an incidence rate of 0.0028 to $0.55 \%$ and complications associated with metastasis. The patient underwent a course of radiotherapy as surgery in this case was contraindicated due to history of excision of the parotid tail.

Risk factors for developing BCCs include intermittent sun exposure, ionising radiation, genetic predisposition and immunosuppression. Metastases occurs via the lymphatic system or haematogenously. Surgery is the main form of treatment and aims to be curative.

Keywords: Basal cell carcinoma, chemotherapy, metastasis, parotid gland, radiotherapy.

Published Online: October 30, 2020

ISSN: 2593-8339

DOI: $10.24018 /$ ejmed.2020.2.5.522

H. Chaudhry*

Oral and Maxillofacial Surgery, Wythenshawe Hospital, Manchester Foundation Trust, UK.

(e-mail: hiba.chaudhry1@nhs.net)

\section{Doumpiotis}

Oral and Maxillofacial Surgery, Wythenshawe Hospital, Manchester Foundation Trust, UK

(e-mail: ddoumpiotis@nhs.net)

*Corresponding Author

\section{INTRODUCTION}

Basal cell carcinomas (BCCs) are malignant neoplasms of the epidermal layer of the skin [1]. BCCs are the most common form of skin cancer accounting for $80 \%$ of nonmelanoma skin cancers [2]. Metastases of BCCs is a rare phenomenon and incident rates account for 0.0028 to $0.55 \%$ of all cases [1]. The majority of metastatic BCCs originate from the head and neck and can disseminate haematogenously or lymphatically with common metastasise to the lungs and regional lymph nodes [3]. Secondary malignant tumours should have a similar histopathological appearance and features to the primary cutaneous lesion [4].

\section{CASE Report}

An 83 year old gentleman presented with a history of $\mathrm{BCC}$ of the right pre-auricular area. A biopsy of the lesion was taken and diagnosed the BCC as an infiltrative subtype. The patient underwent surgery for excision of the lesion and a transposition flap raised from the right posterior auricular to close the defect. Histology confirmed that the BCC had been completely excised but the clear margin was less than
$1 \mathrm{~mm}$. Histology also confirmed that the BCC had invaded down to Clark Level V with no evidence of perineural or lymphovascular invasion. Re-excision was offered to the patient, but the patient chose conservative management with ultrasound every 3 months. The patient was reviewed closely following this period and a lump was noted in the region of the right parotid tail on ultrasound imaging. Clinical examination revealed this to be a $1 \times 1 \mathrm{~cm}$ soft mobile lump at the right parotid tail. Magnetic resonance imaging (MRI) showed a $15 \mathrm{~mm}$ mass in the tail of the right parotid gland on T2 weighted images (Fig. 1) which had not changed significantly in size following ultrasound 3 months prior.

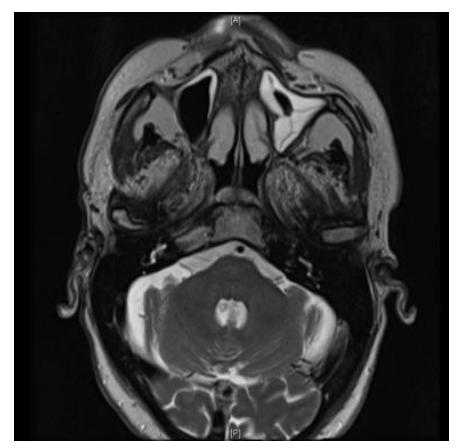

Fig. 1. T2 weighted imaging showing irregular mass $15 \mathrm{~mm}$ in diameter in the tail of the right parotid gland. 
Short T1-inversion recovery (STIR) imaging (Fig. 2) highlighted isointensity to surrounding parotid tissue with signal characteristics not typical of a pleomorphic salivary adenoma or Warthin's tumour.

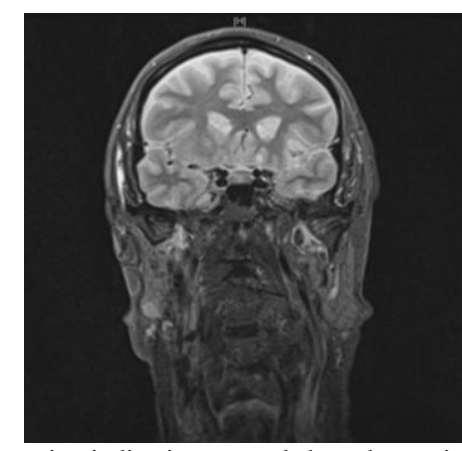

Fig. 2. STIR imaging indicating an oval shaped mass in the right tail of parotid gland.

Fine needle aspiration (FNA) of this lump was inconclusive. The patient was advised to undergo surgical excision of this lump as malignancy could not be excluded at this stage. The patient underwent excision of the tail of the right parotid gland 9 months following initial surgery. Histology of the secondary lesion revealed the presence of basaloid squamous cells with positivity for p40 and CK5/6. Additionally, positivity of MOC31 supported similarity of profile between the secondary tumour and the previous BCC of the right cheek.

Following confirmation of metastases, the patient was referred to the Maxillofacial oncology multidisciplinary team and, in the patient's, best interests, right parotidectomy with preservation of the facial nerve was recommended followed by adjuvant radiotherapy.

\section{DISCUSSION}

BCCs are the most common type of skin cancer in Caucasians and typically occur in areas of the skin exposed to UV light. Evidence also supports intermittent sun exposure including the use of sun beds, occupational exposure and previous episodes of sunburn as additional risk factors [5]. However, 20\% of BCCs occur on areas of the skin not exposed to the UV light and risk factors can include exposure to ionizing radiation, immunosuppression and genetic abnormalities. A number of genetic conditions can predispose to the development of BCCs including Gorlin syndrome and xeroderma pigmentosum [5]. The Fitzpatrick skin type is a reliable indicator of those individuals who are more at risk of developing BCCs, with Fitzpatrick Type I and II having the highest risk.

BCCs often clinically present as poorly defined plaques which may be indurated. They may also form crusts or ulcerations and patients often give a history of spontaneous bleeding and resolution. Clinical features which can indicate high risk include histological subtype, size of tumour and infiltrative growth [6]. Over 26 subtypes of BCC have been reported in the literature; the most frequently occurring are nodular, superficial and morpheaform/ infiltrative types (Table 1).
TABLE 1: THE ABOVE TABLE DESCRIBES FOUR OF THE MOST COMMON SUBTYPES OF BCC [7]. CROWSON DESCRIBES BCCS BEING CLASSIFIED AS INDOLENT-GROWTH OR AGGRESSIVE-GROWTH. INDOLENT BCCS INCLUDE SUPERFICIAL AND NODULAR WHILE MORPHEAFORM AND INFILTRATIVE FALL INTO THE AGGRESSIVE CATEGORY.

\begin{tabular}{ll}
\hline BCC Subtype & Presentation of BCC \\
\hline Superficial & Proliferation of atypical basaloid cells situated \\
& parallel to epidermal surface. May occupy the \\
& hair follicle. Band of lymphoid infiltrate may \\
& be present. \\
& Most common form of BCCs. Characteristic \\
& translucent nodule with rolled borders and \\
& telangiectasia. Characterized by small or large \\
& nests of basaloid cells in the papillary or \\
& reticular dermis. \\
& Also known as sclerosing. Characterized by \\
& basaloid cell columns one to two cells thick. \\
& Poorly demarcated margins extending into \\
& reticular dermis and into subcutaneous tissue. \\
& Often co-exist with other variants of BCC. \\
Morpheaform & Clinically have whitish-yellow depressions. \\
& Invasion into surrounding structures including \\
& perineural invasion must be excluded. \\
& Irregular sized and shaped islands of cells \\
showing high levels of mitotic activity. Poorly \\
demarcated and often show extension into \\
adjacent structures. Perineural invasion is a \\
possible risk and appearance is including flat \\
plaques with whitish-yellow central \\
depressions
\end{tabular}

Metastatic BCCs (mBCC) demonstrate a rare occurrence of a common disease. The most common sites of metastatic spread are the lymph nodes and lungs [2]. Lattes and Kessler in 1951 defined their criteria for the diagnosis of mBCCs [8]. These stated that:

a. Primary tumour must originate from the skin,

b. Metastasis must be at a distant site from the primary tumour and not a primary extension,

c. Histological analysis must demonstrate similar subtype to that of the primary tumour and be consistent with BCC with no squamous component.

Diagnosis is confirmed by biopsy and full histological investigation of the metastatic lesion and compared to the initial tumour. Risk factors for metastasis are reported to include older age, neglected and untreated BCCs, immunodeficiency and a large primary tumour (over $6 \mathrm{~cm}$ ) [9]. Histopathological risk factors have been identified as increased depth of invasion, perineural invasion, loss of peripheral palisading of cells and a lack of local inflammatory cells [9]. BCCs which have infiltrated into Clark Level IV are considered to be high risk for developing metastases [10].

If only regional lymph nodes or local spread have been observed, surgery with or without radiotherapy may be curative [2]. The aim of surgery is curative treatment with a healthy margin. Guidelines by the British Association of Dermatologists state small well-defined BCCs can be excised with a margin of $3 \mathrm{~mm}$ which carries an $85 \%$ success rate or $4-5 \mathrm{~mm}$ will carry a clearance rate of $95 \%$ [11]. Mohs micrographic surgery which was pioneered in the 1940s is the process of staged resection and examination of horizontal sections to ensure no residual pathology remains in the excised tissue. Using this surgical method allows resection of involved margins before surgical closure. 


\section{CONCLUSION}

Disseminated BCC is a rare occurrence but carries a poor prognosis. The possibility of metastasis should be considered in high risk patients as early diagnosis and treatment can prevent further morbidity and mortality. However due to the small number of reported cases and the absence of clinical trials to investigate treatment, evidence regarding treatment remains limited.

\section{ACKNOWLEDGMENTS AND DISClOSURE STATEMENTS}

The authors report no conflicts of interest related to this study.

\section{REFERENCES}

[1] Lau J, Guminski A, Gill A, Veivers D. Metastatic basal cell carcinoma: a review of six cases. Australian Journal of Otolaryngology [Internet]. 2018 Sep 18 [cited 2019 Dec 15];1(0). Available from: http://www.theajo.com/article/view/4091.

[2] Piva de Freitas P, Senna CG, Tabai M, Chone CT, Altemani A. Metastatic Basal Cell Carcinoma: A Rare Manifestation of a Common Disease. Case Rep Med [Internet]. 2017 [cited 2019 Dec 15];2017. Available from: https://www.ncbi.nlm.nih.gov/pmc/articles/PMC5723960/.

[3] Robinson JK, Dahiya M. Basal Cell Carcinoma With Pulmonary and Lymph Node Metastasis Causing Death. Arch Dermatol. 2003 May $1 ; 139(5): 643-8$.

[4] Mehta KS, Mahajan VK, Chauhan PS, Sharma AL, Sharma V, Abhinav C, et al. Metastatic Basal Cell Carcinoma: A Biological Continuum of Basal Cell Carcinoma? Case Reports in Dermatological Medicine. 2012;2012:1-4.

[5] Cameron MC, Lee E, Hibler BP, Barker CA, Mori S, Cordova M, et al. Basal cell carcinoma: Epidemiology; pathophysiology; clinical and histological subtypes; and disease associations. Journal of the American Academy of Dermatology. 2019 Feb 1;80(2):303-17.

[6] Puig S, Berrocal A. Management of high-risk and advanced basal cell carcinoma. Clin Transl Oncol. 2015;17(7):497-503.

[7] Crowson AN. Basal cell carcinoma: biology, morphology and clinical implications. Mod Pathol. 2006 Feb;19 Suppl 2:S127-147.

[8] Lattes R, Kessler RW. Metastasizing basal-cell epithelioma of the skin. Report of two cases. Cancer. 1951;4(4):866-78.

[9] Ionescu DN, Arida M, Jukic DM. Metastatic Basal Cell Carcinoma: Four Case Reports, Review of Literature, and Immunohistochemical Evaluation. Archives of Pathology \& Laboratory Medicine. 2006 Jan 1;130(1):45-51.

[10] Chung S. Basal Cell Carcinoma. Arch Plast Surg. 2012 Mar;39(2):166-70.

[11] Telfer NR, Colver GB, Morton CA. Guidelines for the management of basal cell carcinoma. British Journal of Dermatology. 2008;159(1):35-48. 